\title{
MAGNIFY NETWORK LIFETIME IN WSN BY Reducing Data Aggregation Distance OF WEAK NODES
}

\author{
Prashant Katoch $^{1}$ and Manik Gupta ${ }^{2}$ \\ ${ }^{1}$ Department of Computer Engineering, Chitkara University, Barotiwala \\ ${ }^{2}$ District Solan, Himachal Pradesh, India
}

\begin{abstract}
Energy efficient protocols have always played a vital role in conservation of energy in Wireless Sensor Network. One of the major introduced protocols is LEACH a cluster based protocol. To improve its performance, an algorithm named as Maximizing the Network Lifetime of Clustered-based WSN Using Probability of Residual Energy is introduced. This protocol improved the Cluster Head selection process of LEACH by using the concept of residual energy. In the proposed paper, further improvement is done by enhancing the data transmission process. This process heightens the lifetime of the nodes having very less energy left by reducing their data transmission distance. Implemented results in MATLAB shows increase in the stability and lifetime of the network.
\end{abstract}

\section{KEYWORDS}

WSN, FND, LEACH, modified algorithm, data transmission

\section{INTRODUCTION}

A collection of large number of wireless sensor nodes having capability of sensing and transmitting data form a WSN. These nodes having limited capabilities of storage, processing and power are deployed in a large area at distant places. These nodes collect the data by sensing process and transmit it further to the base station. Sensor nodes could be of different categories infrared, radar, seismic and thermal visual [1]. For different environmental and physical conditions like heat, pressure, temperature surveillance and monitoring of different day to day activities of our own environment like traffic, home or office monitoring, WSN is used [2] [3].

Nodes have limited power storage and change of batteries is unhandy, so energy and communication bandwidth are the two major problems that we face [4]. One time transmission of data by all the nodes to the BS completes one round. With time and rounds energy of the nodes also starts to decrease. Nodes which are having same energy level in the beginning of the first round do not have same energy level as the rounds pass, this is because they consume different amount of energy depending upon the factors like distance and cluster hierarchies [5]. Due to small battery power and more energy consumption nodes start dying, to avoid it various energy efficient protocols are utilized. In cluster based approach, some nodes are chosen as $\mathrm{CHs}$ and others work as member nodes [6]. Data collected by member nodes by sensing is trasmitted to their $\mathrm{CHs}$ and $\mathrm{CHs}$ will send that data to BS after aggregation [7]. Two types of cluster based 
routing protocols are used, one is homogenous and other is hetrogeneous. In homogeneous approach, the sensors nodes are having same capabilities and hardware configurations [8]. In hetrogeneous approach, sensor nodes are having different capabilities, for example, few nodes are given high capabilities [8]. In hetrogenity, some nodes have more physical capabilities like higher bandwidth, can cover longer distance network than others and their batteries are replacable [9]. Our approach in this paper is based on the homogenity. One of the well known approach in homogenity is Low-Energy Adaptive Clustering Hierarchy(LEACH) [10].. To improve the CH selection process in this clustering protocol an approach called Maximizing the Network Lifetime of Cluster-based WSN Using Probability of Residual Energy was introduced [11]. In this paper, we have introduced an approach to improve the network lifetime and stability of this protocol by reducing the data transmission distance of the depleting nodes by enhancing the transmission way of the network.

The rest of the paper is organized as follows. Section 2 describes the related work. Section 3 demonstrates the networking model and working of our protocol. In section 4 the simulation results are shown and comparison of our protocol with LEACH and MODIFIED ALGORITHM is performed. In the section 5, paper is concluded.

\section{RELATED WORK}

\subsection{LEACH}

Leach is a multiple cluster based protocol in which every cluster has a $\mathrm{CH}$ node. All the nodes of cluster send their sensed data to this node and this node aggregate that data and send to BS hence reducing the communication overhead of all the nodes.

LEACH is a self-organizing, adaptive clustering protocol that uses randomization to distribute the energy load evenly among the sensors in the network [10]. With every round $\mathrm{CH}$ changes depending upon the remaining energy because if one node remains the $\mathrm{CH}$ then its energy will refrain quickly. So LEACH is divided into two phases.

One is setup phase, in which $\mathrm{CH}$ is selected by the nodes using probability function. Second phase is steady state phase, in which all the nodes send the data to their respective $\mathrm{CH}$ and then $\mathrm{CH}$ send to BS.

Election of the $\mathrm{CH}$ is done by selecting a number between 0 and 1 which should be less than the threshold $T(n)$. Threshold formula $T(n)$ is calculated as [10].

$$
T(n)=\left\{\begin{array}{l}
\frac{p}{1-p\left(r \bmod \frac{1}{p}\right.}, \text { if } n \in G \\
0 \quad \text { otherwise }
\end{array}\right.
$$

Where $\mathrm{p}$ is the desired number of $\mathrm{CHs}, \mathrm{r}$ is the current round and $\mathrm{G}$ is the set of nodes that has not been $\mathrm{CH}$ in $1 / \mathrm{p}$ rounds. Within $1 / \mathrm{p}$ rounds, every node will become $\mathrm{CH}$. In the beginning of the first round, each node has the probability $\mathrm{p}$ to become $\mathrm{CH}$ and the node which has become $\mathrm{CH}$ in the first round, that node cannot be a $\mathrm{CH}$ in next $1 / \mathrm{p}$ rounds. Every node's probability to become a $\mathrm{CH}$ increases. All the nodes would have been $\mathrm{CH}$ after 1/p rounds [10].

After the clusters creation and fixation of TDMA schedule the transmission of data packets begin. Nodes send data to their assigned CHs and at given TDMA slot. Radio of sensor node will only be on at their respective time slots but receiver of the $\mathrm{CH}$ will always remain $\mathrm{ON}$. After the end of this phase setup phase will begin for the next round. 


\subsection{Modified Algorithm for CH Selection}

This algorithm is developed to overcome the limitations of LEACH protocol. In LEACH protocol $\mathrm{CH}$ is selected on the basis of initial energy but after few rounds the energy of the nodes become uneven then $\mathrm{CH}$ selection process is not much of a use. To overcome that disadvantage, this protocol has introduced the selection of the $\mathrm{CH}$ on the basis of node's residual energy [11]. The algorithm has been divided in three phases, first is setup phase, second is steady state phase and third is pre set-up phase.

\subsubsection{CH selection}

$\mathrm{CH}$ selection process involves residual energy of each node. Residual energy is the remaining energy of a node that can be used for communication in the upcoming rounds. When residual energy is zero then node is considered as dead. Node's energy will decrease as the rounds progress. Residual energy is an efficient parameter to select the $\mathrm{CH}$ [12]. Now probability of the node to become a $\mathrm{CH}$ is now depends upon its energy which will help in the better selection of $\mathrm{CH}$ even the uneven residual energies of nodes. So nodes having high residual energy will have more probability of becoming the $\mathrm{CH}$ than the lower residual energy nodes. This helps in stabilizing the network by ensuring that nodes can die almost at same time. Set the probability of $\mathrm{CH}$ selection as a function of node's residual energy relative to the aggregate remaining energy [11]. Threshold energy is calculated as

$$
T(n)=\left\{\begin{array}{cl}
\frac{p_{\text {opt }}}{1-p_{\text {opt }} *\left(r \bmod \frac{1}{p_{\text {opt }}}\right)} \cdot \frac{E_{\text {residual }}(t)}{E_{\text {total }}(t)} \text { if } n \in G \\
0 \quad \text { otherwise }
\end{array}\right.
$$

Where $p_{\text {opt }}$ is calculated as $k_{o p} / n r, p_{\text {opt }}$ is the $\mathrm{CH}$ selection probability, $k_{\text {opt }}$ is the optimum number of clusters in the current round, $n$ is number of nodes and $r$ is number of rounds. $G$ is the set of nodes which have not been $\mathrm{CH}$ last $1 / p_{\text {opt }}$ rounds, $E_{\text {residual }}$ is the residual energy of the node and $E_{\text {total }}$ is the total energy of the entire network. $E_{\text {total }}(t)$ is calculated as [11]

$$
E_{\text {total }}(t)=\sum_{n=1}^{N} E_{\text {residual }}(t)
$$

\subsubsection{Setup phase}

In the beginning of this phase, the probability function $p_{n}$ is randomly calculated and then with its help threshold value $T(n)$ is calculated. After selection of the $\mathrm{CH}$ will make a link with its associated nodes and TDMA schedule is prepared for all the nodes.

\subsubsection{Steady state phase}

Its steady state phase is similar to LEACH. In this state the nodes will sense and transmit the data to its respective $\mathrm{CH}$ in its assigned TDMA slot. Every node will send data that it has sensed, its current energy and its ID. $\mathrm{CH}$ then aggregate the all the energy and send to the BS. 


\subsubsection{Next round pre set-up phase}

In this phase, the $\mathrm{BS}$ receive all the remaining energy level values of all the nodes from their respective $\mathrm{CHs}$ and then calculate the $E_{\text {total }}$ that is total residual energy of the network and then send it back to $\mathrm{CH}$. Each node receive this value from their respective $\mathrm{CHs}$ and each node save this value for the calculation of threshold in the next round which completes one round of the communication [11].

\section{PROPOSED PROTOCOL}

In this section we will discuss the working of our protocol in WSN. Both LEACH[10] and modified LEACH[11] algorithms have not provided a way to reduce the energy consumption of weak or depleting nodes. In this paper, we have discussed a way to reduce the energy consumption of the weak nodes in the cluster so that they can last for more rounds. It will also make the network more even and hence the cluster head selection will also be more reasonable. In the modified algorithm all the nodes in the cluster send their sensed data to their respective $\mathrm{CH}$ and then $\mathrm{CH}$ will forward to BS. All the nodes in a cluster excluding $\mathrm{CH}$ are treated same despite of their uneven remaining energies. All the nodes have to transmit the data to $\mathrm{CH}$, so sometimes nodes are far away from their CHs. This is a problem for the weak nodes as they have to send the data to a distant place and hence more energy is required for transmission [13]. This leads to the early death of these nodes. Hence stability of network reduces. Hence to avoid this we have developed an algorithm to efficiently transmit the data. We introduced a transmission algorithm in setup phase whereas steady phase and $\mathrm{CH}$ selection phase will remain same.

\subsection{Setup phase}

In the beginning of this round, random probability $\left(p_{n}\right)$ by each node is generated and then the threshold value $T(n)$ is calculated by using node's residual energy and total residual energy of the network. Threshold value is calculated as [11]

$$
T(n)=\left\{\begin{array}{cl}
\frac{p_{\text {opt }}}{1-p_{\text {opt }} *\left(r \bmod \frac{1}{p_{\text {opt }}}\right)} \cdot \frac{E_{\text {residual }}(t)}{E_{\text {total }}(t)} \text { if } n \in G \\
0 \quad \text { otherwise }
\end{array}\right.
$$

Where $E_{\text {residual }}$ is the residual energy of the node and $E_{\text {total }}$ is the aggregation of total energy left in the network. $p_{\text {opt }}$ is optimum $\mathrm{CH}$ selection probability, $k_{\text {opt }}$ is the optimum number of $\mathrm{CH}$ and $\mathrm{G}$ is the set of the nodes that never have been cluster $\mathrm{CH}$ in last $1 / p_{\text {opt }}$ rounds. $E_{\text {total }}(t)$ is calculated as[11]

$$
E_{\text {total }}(t)=\sum_{n=1}^{N} E_{\text {residual }}(t)
$$

Nodes in the network have same initial energy but after few rounds, energy become uneven and hence some nodes which are having very less amount of energy left cannot afford a long distant $\mathrm{CH}$ because they have to spend more energy for that. So we have introduced an efficient way for those nodes to transmit their data. Nodes have been divided into three categories. 
1) $\mathrm{CH}$ node

2) Normal node

3) Weak node

To make sure some nodes in the network do not die early, we have to concentrate on the diminishing energy of those nodes and have to find a way to stop them dying early as compared to their companion nodes which will help in making network more stable, cluster making and easy selection of CHs in network. So, survival of these nodes plays a vital role in the network. We call these nodes weak nodes. To evaluate which node has to be categorized as weak nodes, we have built a threshold function.

$\mathrm{CH}$ node is selected at the beginning of round using a threshold function. The nodes which are not $\mathrm{CH}$ and have energy more than the weak node threshold function $\left(T_{w}\right)$ can be called as normal nodes. Weak nodes are those having energy less than the weak node threshold function $\left(T_{w}\right)$ and hence are near of dying. Weak node threshold function $\left(T_{w}\right)$ can be calculated as

$$
T_{w}=E_{\text {initial }} / 10
$$

$E_{\text {initial }}$ is the initial energy of a node. This equation counts the 10 per cent of the initial energy of the network. So, nodes having energy less than 10 per cent of the initial node energy are considered as weak nodes.

\subsubsection{Data Transmission Algorithm (CH, DT, BS, WN, NN, N)}

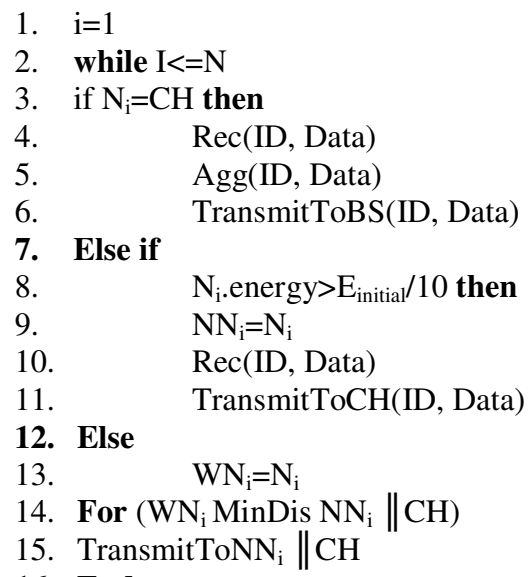

Where $\mathrm{CH}$ is cluster head, DT is data transmission, $\mathrm{WN}$ is weak node, $\mathrm{NN}$ is normal node, $\mathrm{E}_{\text {initial }}$ is initial energy of the node, BS is base station, $\mathrm{N}_{\mathrm{i}}$ is set of all the nodes.

In this algorithm, first of all we check whether the node is $\mathrm{CH}$ or not, if it is, then the data transmission (DT) will be done to BS. If node is not $\mathrm{CH}$ then we will check whether the node has energy more than 10 per cent of the initial node energy or not. The node which satisfies this condition will we considered as a normal node (NN) and this node will transmit the data to its respective cluster head. If node has less than 10 per cent of the initial node energy then the node is categorized as a weak node and it will transmit the data to the nearest possible node $\left(\mathrm{N}_{\mathrm{i}}\right)$ whether it is normal node or $\mathrm{CH}$ node. 
Network stability is very important expect of WSN, nodes of network should not have much difference in terms of death time. So, this protocol will help the weaker nodes to remain alive for longer period by reducing their distance of data transmission and hence reducing the amount of energy utilized. So, all nodes will stay alive for longer period, making the network more efficient and stable.

A message is then broadcasted to all the nearby nodes and nodes send request message to join the network and then $\mathrm{CH}$ will make a list of its associated nodes. Every node is then given a timeslot for the transfer of data to $\mathrm{CH}$. That timeslot is called as a TDMA schedule [11] and it will then broadcasted to all the nodes of the cluster.

\subsection{Steady state phase}

In this phase, two main tasks are performed. First is sensing and second is transmission of data. Life of a node also very much depends upon the transmission of data. Energy used in transmission of data normally depends upon things like distance and packet size[14]. CH transmit the data in free-space where as a normal node transmit in multipath, so more energy is required if the data has to be transmitted using free space. We cannot reduce the size of the data but we can reduce the distance that a node's data has to cover before reaching its destination. So we have reduced the distance coverage for the weaker nodes, to increase their lifetime. The weak nodes will transmit to the nearby node and then that node will transmit data to $\mathrm{CH}$ as shown in the setup phase.

\subsection{Next round pre set-up phase}

In this round the $\mathrm{BS}$ aggregate the residual energy of all the remaining nodes and send back the value of $E_{\text {total }}$ to all the $\mathrm{CHs}[11]$. Then $\mathrm{CHs}$ will forward it to their respective cluster nodes. This value will be used in the $\mathrm{CH}$ selection process of the next round.

\section{SIMULATIONS AND RESULTS OF THE PROTOCOL}

\subsection{Simulation Parameters}

Simulation of this protocol has been done using Matlab simulator. We have obtained the outcomes of the protocol after certain number of rounds. We have used same parameters on LEACH, modified algorithm and our protocol to obtain the results. Results have been obtained the network lifetime using the parameter of First Node Dead (FND). FND defines the number of rounds after which the nodes start dying. Energy dissipation model which we have used assume the 50nJ/bit electricity for transmitter and receiver circuitry. To calculate the energy dissipation radio model has been used[10].

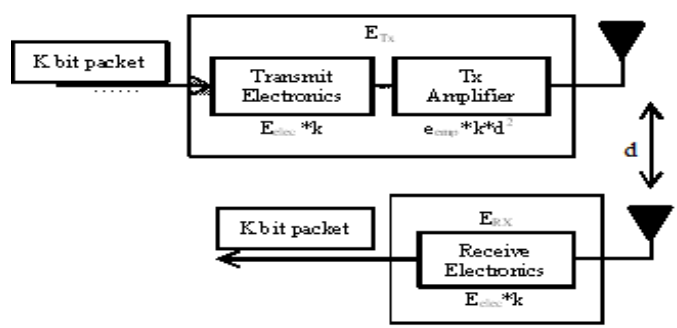

Figure 1. First radio Model[10]. 


\subsubsection{First Node Dead}

First Node Dead (FND) states that after how many rounds first node in the network is dying. FND is an efficient way to calculate network lifetime [15]. It shows how stable and efficient a network is.

Simulation of the protocol in FND shows the better results on both smaller and larger networks in comparison to both LEACH and modified algorithm. Implementation is done using different parameters like variation in network size and number of nodes. Simulation parameters are shown in table 1 .

Table 1.Simulation Parameters

\begin{tabular}{|l|l|}
\hline Parameters & Value \\
\hline Network Size(M x M) & $(100,100),(500,500)$ \\
Base Station & At the centre of network \\
Initial Energy & $.5 \mathrm{~J} / \mathrm{battery} /$ Node \\
Packet Size & $4000 \mathrm{bits}$ \\
Nodes & $100,75,50$ \\
Tx Energy Consumption. & $50 \mathrm{nj} / \mathrm{bit}$ \\
Rx Energy Consumption. & $50 \mathrm{nj} / \mathrm{bit}$ \\
Amplifier energy consumption & $100 \mathrm{pj} / \mathrm{bit} / \mathrm{m}^{2}$ \\
& \\
\hline
\end{tabular}




\subsection{Results and Analysis}

Results have been generated and analysed using tables. Flow chart shows the working of protocol.

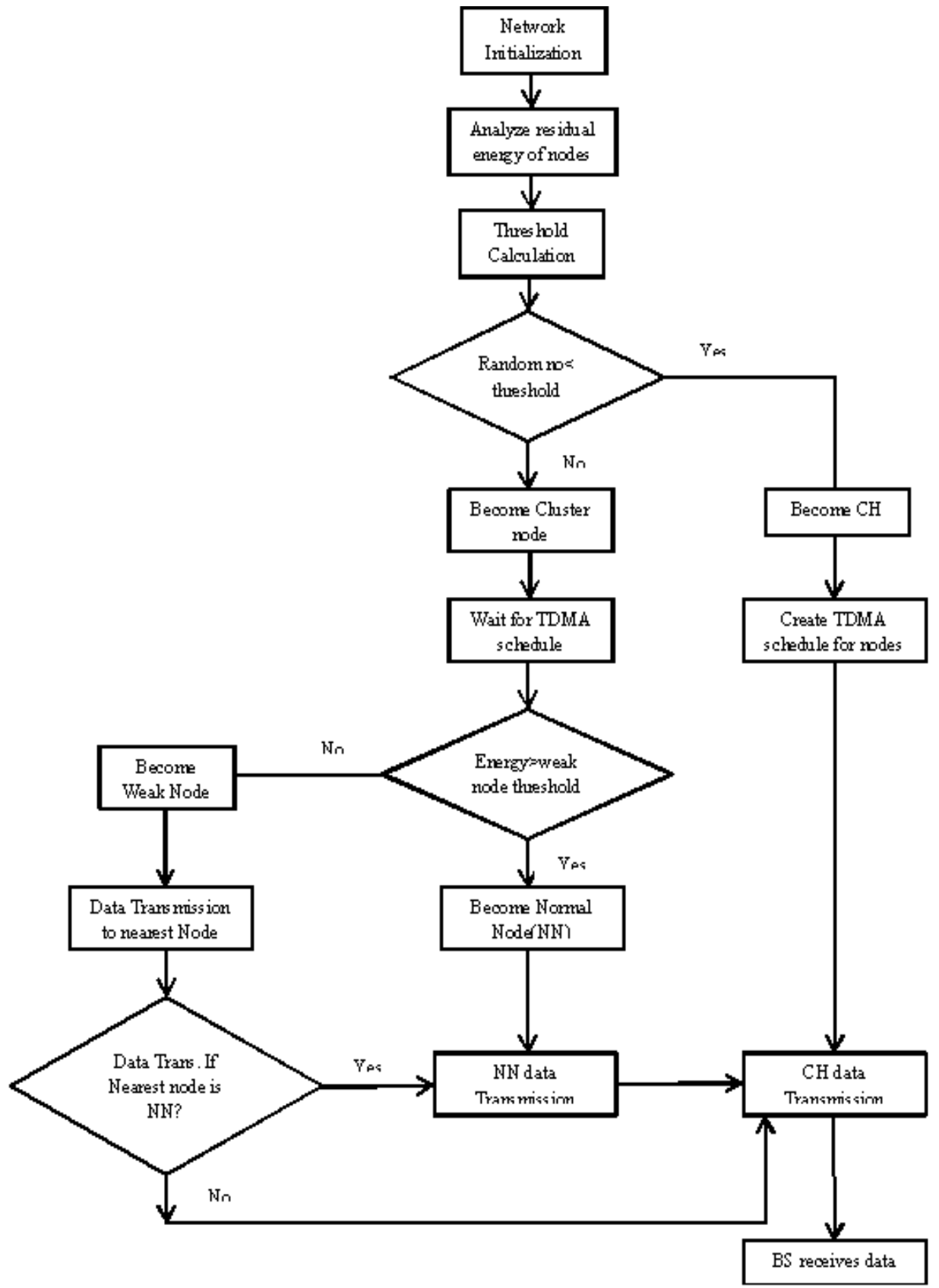

Figure.2. Flow chart of the proposed protocol.

Flow chart in Figure 2. shows how the protocol works and how the transmission gets done. 
International Journal of Wireless \& Mobile Networks (IJWMN) Vol. 7, No. 6, December 2015

\subsubsection{Generated Graphs}

Graphs have been generated by the implementation of these protocols using different parameters.

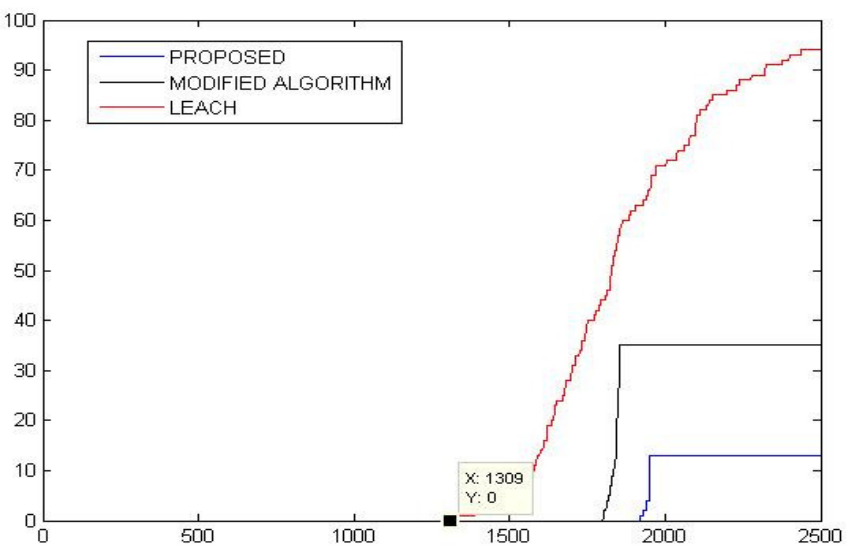

Figure.3. FND for nodes $=100$ and network size $100 \mathrm{~m} \times 100 \mathrm{~m}$.

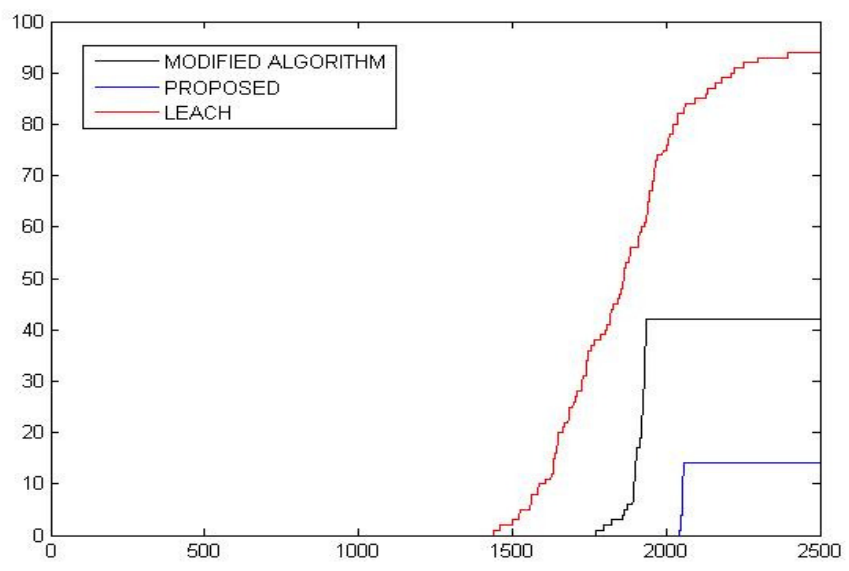

Figure.4. FND for nodes=75 and network size $100 \mathrm{~m} \times 100 \mathrm{~m}$.

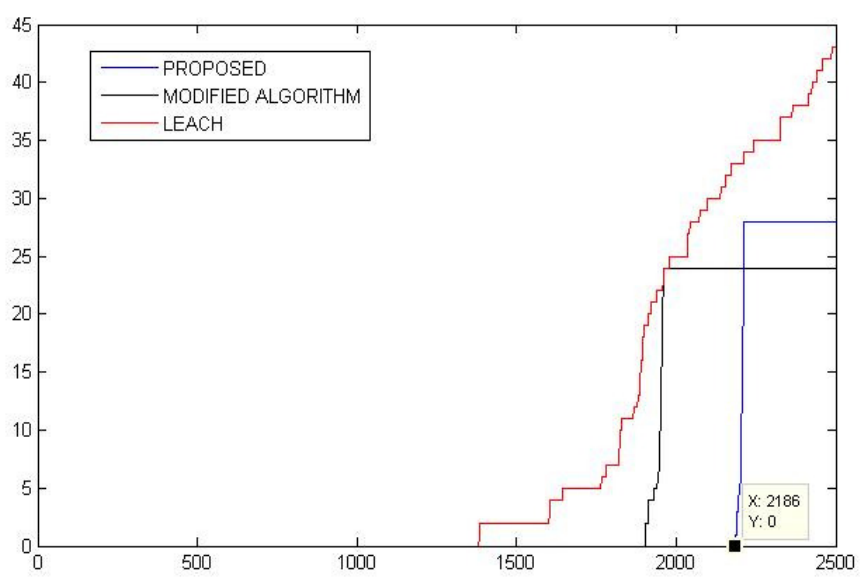

Figure.5. FND for nodes $=50$ and network size $100 \mathrm{~m} \times 100 \mathrm{~m}$ 
Fig.3, fig.4 and fig.5 represent the enhancement of proposed protocol over LEACH and modified algorithm in terms of network lifetime of the small scale network $(100 \mathrm{~m} \times 100 \mathrm{~m})$. As the number of nodes decreases the performance of all the protocols starts enhancing but in all the cases proposed protocol's performance over other two protocols is always better.

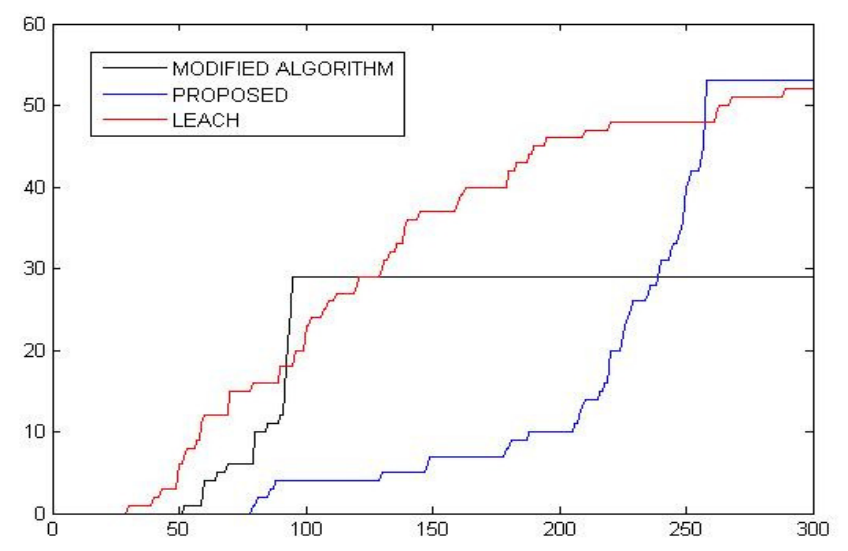

Figure.6. FND for nodes=100 and network size $500 \mathrm{~m} \times 500 \mathrm{~m}$

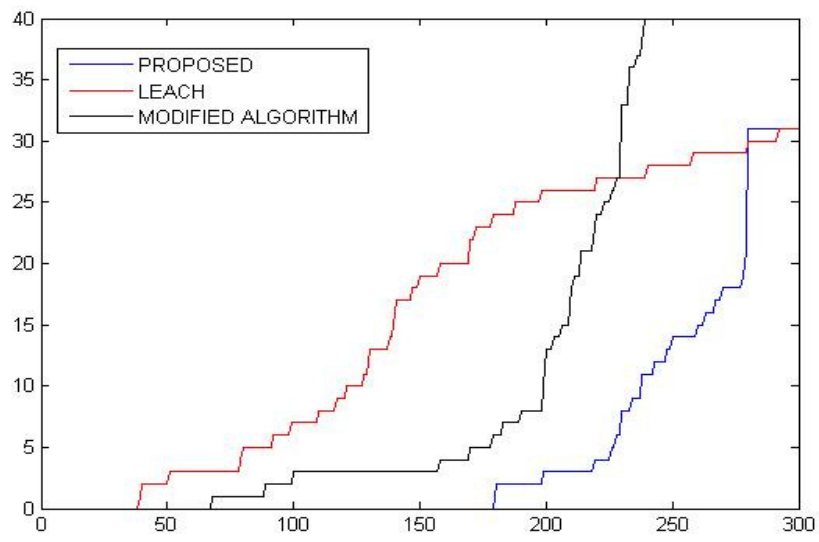

Figure.7. FND for nodes=75 and network size $500 \mathrm{~m} \times 500 \mathrm{~m}$

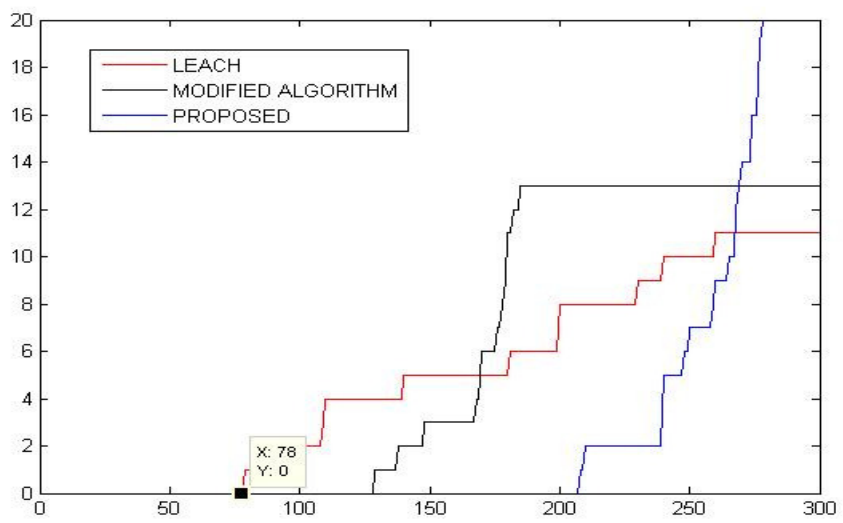

Figure.8. FND for nodes $=50$ and network size $500 \mathrm{~m} \times 500 \mathrm{~m}$ 
Fig.6, fig.7 and fig.8 shows the improvement of lifetime in proposed protocol over both LEACH and Modified algorithm for large scale network $(500 \mathrm{~m}$ x $500 \mathrm{~m})$ using different number of nodes. This variation of parameters is applied to show the adaptability of protocol in different conditions. Improvement in all the three graphs is seen for the proposed protocol. We can see the exact values of the implementation in the table 2 and 3 given below.

Table 2. Results for $100 \mathrm{~m}$ x $100 \mathrm{~m}$ network.

\begin{tabular}{|l|l|l|l|}
\hline No of nodes & $\mathbf{1 0 0}$ & $\mathbf{7 5}$ & $\mathbf{5 0}$ \\
\hline Leach & 1309 & 1398 & 1382 \\
\hline $\begin{array}{l}\text { Modified } \\
\text { Algorithm }\end{array}$ & 1794 & 1816 & 1902 \\
\hline Proposed & 1919 & 1998 & 2187 \\
\hline
\end{tabular}

Table 3. Results for 500m x 500m network.

\begin{tabular}{|l|l|l|l|}
\hline No of nodes & $\mathbf{1 0 0}$ & $\mathbf{7 5}$ & $\mathbf{5 0}$ \\
\hline Leach & 29 & 38 & 78 \\
\hline $\begin{array}{l}\text { Modified } \\
\text { Algorithm }\end{array}$ & 51 & 67 & 129 \\
\hline Proposed & 79 & 179 & 207 \\
\hline
\end{tabular}

We have seen that as the number of nodes increase, the value of FND decreases but still for all different number of nodes our proposed algorithm shows improvement.

\subsubsection{Analysis of results for different parameters}

Result shows how parameters different parameters affect differently on the resultant values of the protocol. As we increase the network size, the network lifetime decreases for all the protocols, which is because of the fact that nodes have to cover less distance in smaller size networks. Network lifetime also got affected by the number of nodes. As we decrease the number of nodes, the network lifetime becomes better. Proposed work shows better results in all the variations of parameters.

The results show different values for varying parameters, we have seen the results in variation of two sizes of the network $100 \mathrm{~m} \times 100 \mathrm{~m}$ and $500 \mathrm{~m} \times 500 \mathrm{~m}$ for 100,75 and 50 nodes. These results show better percentage of improvement for the larger size network and smaller number of nodes. But improvement is there in every case. Better results of protocol shows the adaptability of the protocol in the variation of network size and number of nodes. 


\section{CONCLUSION}

Implementation performed on LEACH, modified algorithm and our protocol using a variety of parameters. We have analysed the results of all of them and have seen that how our protocol improve the results of network lifetime on the basis of FND over the two protocols. We have concluded that increasing the lifetime of weak nodes of network can increase the overall network lifetime of the network. This protocol is much more stable then LEACH and modified algorithm. Implementation performed in the MATLAB has improved the transmission of data over the network. This transmission protocol can be applied in many other $\mathrm{CH}$ selection algorithms and help them improving their performance. It has continuously improved the network lifetime for variety of parameters over modified algorithm and LEACH.

\section{REFERENCES}

[1] Sheetal Mehta Parul Bakaraniya, "K-LEACH: An improved LEACH Protocol for," International Journal of Engineering Trends and Technology, vol. 4, no. 5, may 2013.

[2] A. AbdulMaowjod and H. Mohammed, Q. Ali, ""Simulation and Performance Study of Wireless Sensor Network (WSN) Using MATLAB," Iraq J. Electrical and Electronic Engineering, vol. vol. 7, no. no.2, pp. pp. 112 - 119, 2011.

[3] L.F, ""Smart Environments: Tecnologie, Protocols and Application," in Wireless Sensor Network," 2004.

[4] Anantha P. Chandrakasan,Hari Balakrishnan Wendi B. Heinzelman, "An Application-Specific Protocol Architecture for Wireless Microsensor Networks," IEEE TRANSACTIONS ON WIRELESS COMMUNICATIONS, vol. VOL. 1, no. NO. 4, OCTOBER 2002.

[5] Jeremiah D. Deng, Martin K. Purvis Femi A. Aderohunmu, "A Deterministic Energy-efficient Clustering Protocol," 2011.

[6] Nejah NASRI, Anne WEI, Abdennaceur KACHOURI Salim EL KHEDIRI, "A New Approach for Clustering in Wireless Sensors Networks," International Workshop on Wireless Networks and Energy Saving Techniques, pp. 1180-1185, 2014.

[7] Abdalraheem A. Ijjeh Raed M. Bani Hani, "A Survey on LEACH-Based Energy Aware Protocols for Wireless Sensor Networks," Journal of Communications, vol. Vol. 8, no. No. 3, March 2013.

[8] N. Javaid, A. H. Khan, A. Iqbal, E. Akhtar, M. Ishfaq T. N. Qureshi, "BEENISH: Balanced Energy Efficient Network Integrated," International Workshop on Body Area Sensor Networks, march 2013.

[9] "HETEROGENEOUS CLUSTER BASED ROUTING PROTOCOLS IN WIRELESS SENSOR NETWORK- A SURVEY," International Journal of Research in Advent Technology, vol. Volume 1, no. Issue 4, November 2013.

[10] Anantha Chandrakasan, and Hari Balakrishnan Wendi Rabiner Heinzelman, "Energy-Efficient Communication Protocol forWireless Microsensor Networks," Proceedings of the 33rd Hawaii International Conference on System Sciences, 2000.

[11] Saltihie Zeni Jiwa Abdullah, "Maximizing the Network Lifetime of Clustered-based WSN Using Probability of Residual Energy," IEEE International Conference on Control System,Computing and Engineering, pp. 28 - 30, November 2014.

[12] D. Q. Lu Cheng and W. Wu, An energy efficient weight-clustering algorithm in wireless sensor networks.: Proc. Japan-China Joint Workshop on Frontier of Computer Science and Technology, IEEE Computer Society, 2008.

[13] Kamal Kant, Nitin Gupta Kiran Maraiya, "Wireless Sensor Network: A Review on Data," International Journal of Scientific \& Engineering Research, vol. 2, no. 4, april 2011.

[14] Doan B.Hoang Najmeh Kamyabpour, "Modeling overall energy consumption in Wireless Sensor Networks".

[15] Praveen Kaushik, Jyoti Singhai Sourabh Jain, ENERGY EFFICIENT MAXIMUM LIFETIME ROUTING FOR WIRELESS SENSOR NETWORK. Bhopal, india: International Journal Of Advanced Smart Sensor Network Systems ( IJASSN ), January 2012, vol. 2. 